Gene, 152 (1995) 247-251

(C) 1995 Elsevier Science B.V. All rights reserved. 0378-1119/95/\$9.50

\title{
RTR: a new member of the nuclear receptor superfamily that is highly expressed in murine testis
}

\author{
(Orphan receptor; RXR; spermatogenesis; transcription factor; spermatids; PCR)
}

\author{
Takahisa Hirose ${ }^{\mathrm{a}}$, Deborah A. O’Brien ${ }^{\mathrm{b}}$ and Anton M. Jetten ${ }^{\mathrm{a}}$ \\ ${ }^{2}$ Cell Biology Section, Laboratory of Pulmonary Pathobiology, National Institute of Environmental Health Sciences, National Institutes of Health, \\ Research Triangle Park, NC 27709, USA; and ${ }^{\mathbf{b}}$ Laboratories for Reproductive Biology and Department of Pediatrics and Cell Biology and Anatomy, \\ University of North Carolina, Chapel Hill, NC 27599, USA
}

Received by C.M. Kane: 7 June 1994; Revised/Accepted: 17 August 1994; Received at publishers: 22 September 1994

SUMMARY

We have identified and cloned a novel member of the nuclear receptor superfamily from murine testis, referred to as retinoid receptor-related testis-associated receptor or RTR. Degenerate PCR primers homologous to two conserved regions of the DNA-binding domain of members of this superfamily were employed to identify this gene. The aminoacid sequence of RTR is most closely related to that of the mouse RXRs with an overall identity of 32-34\%; the highest similarity $(61 \%)$ is observed in the DNA-binding domain. Northern blot analysis using RNA from multiple tissues showed that RTR is predominantly expressed in the testis. Northern blot analysis using RNA from different testicular cell types showed that RTR mRNA is not expressed in early germ cells or Sertoli cells but is most abundant in round spermatids. Our observations suggest that this putative transcription factor plays a role in the regulation of gene expression particularly during the post-meiotic phase of spermatogenesis.

\section{INTRODUCTION}

The nuclear receptors comprise a large family of ligand-dependent transcriptional factors that regulate gene expression during specific developmental processes, cell growth and differentiation (Yamamoto et al., 1985).

Correspondence to: Dr. A.M. Jetten, Cell Biology Section, National Institute of Environmental Health Sciences, National Institutes of Health, Research Triangle Park, NC 27709, USA. Tel. (1-919) 541-2768; Fax (1-919) 541-4133; e-mail: jetten@niehs.nih.gov

Abbreviations: aa, amino acid(s); COUP-TF, chicken ovalbumin upstream promoter transcription factor; $\mathrm{Mu}-\mathrm{MLV}$, murine Moloney leukemia virus; nt, nucleotide(s); PCR, polymerase chain reaction; PPAR, peroxisome proliferator-activated receptor; RAR, retinoic acid receptor; RTR, retinoid receptor-related testis-associated receptor; $R T R$, gene (DNA, RNA) encoding RTR; RXR, retinoid X receptor.
This family includes the steroid and thyroid hormone receptors, retinoid receptors, peroxisome proliferatoractivated receptors and several orphan receptors for which the ligand has not yet been identified. Members of this nuclear receptor family share several structural and functional characteristics (Green and Chambon, 1988; Evans, 1988). This superfamily appears to be defined in particular by the DNA-binding domain which is the most highly conserved among the family members and contains two zinc-finger motifs (Laudet et al., 1992).

In this study, we report the isolation and partial characterization of an additional member of this nuclear receptor superfamily which we refer to as RTR. We demonstrate that this receptor is highly expressed in the testis and particularly in round spermatids, suggesting a role for RTR in the regulation of gene expression during the post-meiotic phase of spermatogenesis. 
GAATTCCTCACGGGCATGTGCGTGGCAGCGGCGCGTGACGTCAGAGGAGGGAGCTGGC 58 CAGTGCTGAGGGGGCTCGGCGCGGAGGGGCGCGGAGCCGGGCGGCTCAGGGGCCCAGAGA GTCACGACTCCCCCGAGCCGCGCCTCCCCGCGCCTCGGCCCGCCGAGTCCCCGGGTCTCP GTGCGGCGGCCGAGAGCCT GCCGGCCCCTGACAGCCCCCTCCCCCCGIGGAAGACCAGGA CACGCCGCCGGCTCTCGGACGGCCGGGGACTGTCGGGGGaGGBGGGCACCITCTGGTCC: 238 CGCCGCATGGAGCGGGACGAACGGCCACCTAGCGGAGGGGGAGGCGGCGGGGGCTCGGCG 298

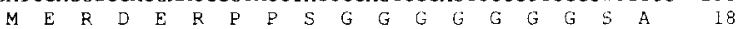
GGGTTCCTGGAGCCGCCCGCCGCGCTCCCTCCGCCGCCGCGCAACGGTTTCTGTCAGGAT 358

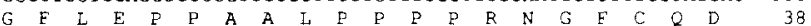
GAATTGGCAGAGCTTGATCCAGGCACTAATGGAGAGACTGACAGTTTAACACTTGGCCAA 418

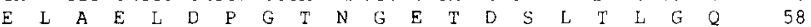
GGCCATATACCTGTTTCCGTCCCAGATGATCGAGCTGAACAACGACCTGTCTCATCTGT 478 $\begin{array}{lllllllllllllllllllll} & H & I & P & V & S & V & P & D & D & R & A & E & Q & R & T & Q & L & I & C & 78\end{array}$ GGGGACCGCGCTACGGGCTTGCACTATGGGATCATCTCCTGCGAGGGCTGCAAGGGGTTT 538 G D B A I G L H Y G I I S C E G C K E E F 98 TTCAAGAGGAGCATTTGCAACAAACGGGTGTATCGGTGCAGTCGTGACAAGAACTGTGTC 598

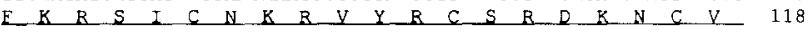
ATGTCCCGGAAGCAGAGGAACAGATGTCAGTACTGCCGCCTGCTCAAGTGTCTCCAGATG 658

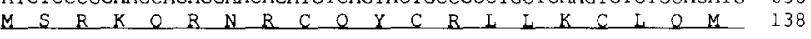
GGCATGAACAGGAAGGCTATCAGAGAAGATGGCATGCCTGGAGGCCGGAACAAGAGCATT 718 $\begin{array}{lllllllllllllllllllll}G & M & N & R & K & A & I & R & E & D & G & M & P & G & G & R & N & K & S & I & 158\end{array}$ GGACCAGTCCAGATATCAGAAGAGGAATTGAAaGAATCATGTCTGGACAGGAGTTTGAG 778 $\begin{array}{llllllllllllllllllllll}G & P & V & Q & \text { I } & S & E & E & E & I & E & R & I & M & S & G & Q & E & F & E & 178\end{array}$ GAAGAAGCCAATCACTGGAGCAACCATGGTGACAGCGACCACAGTTCCCCTGGGAACAGG 838 $\begin{array}{lllllllllllllllllllll}\text { E } & E & A & N & H & \text { W } & S & N & H & G & D & S & D & H & S & S & P & G & N & R & 198\end{array}$ GCTTCAGAGAGCAACCAGCCCTCACCAGGCTCCACACTATCATCCAGTAGGTCTGTGGAa 898 $\begin{array}{llllllllllllllllllllll}A & S & E & S & N & Q & \text { P } & S & P & G & S & T & L & S & S & S & R & S & V & \Xi & 218\end{array}$ CTAAATGGATTCATGGCATTCAGGGATCAGTACATGGGGATGTCAGTGCCTCCACATTAT 958

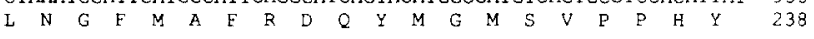
CAATACATACCACACCTTTTTAGCTATTCTGGCCACTCACCACTTTTGCCCCCACAAGCT 1018 $\begin{array}{lllllllllllllllllllll}Q & Y & \text { I } & \text { P } & \text { H } & \text { L } & \text { F } & \text { S } & \text { Y } & \text { S } & G & \text { H } & \text { S } & \text { P } & \text { L } & \text { L } & \text { P } & \text { Q } & Q & \text { A } & 258\end{array}$ CGAAGCCTGGACCCTCAGTCCTACAGTCTGATTCATCAGCTGATGTCAGCCGAAGACCTG 1078 $\begin{array}{lllllllllllllllllllllll}\mathrm{R} & \mathrm{S} & \mathrm{L} & \mathrm{D} & \mathrm{P} & \mathrm{Q} & \mathrm{S} & \mathrm{Y} & \mathrm{S} & \mathrm{L} & \mathrm{I} & \mathrm{H} & \mathrm{Q} & \mathrm{L} & \mathrm{M} & \mathrm{S} & \mathrm{A} & \mathrm{E} & \mathrm{D} & \mathrm{L} & 278\end{array}$ GAGCCATTGGGCACACCTATGTTGATTGAaGATGGGTATGCTGTGACACAGGCAGAACTG 1138

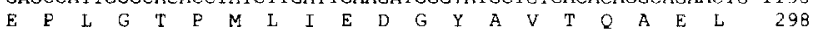
TTTGCTCTGCTTTGCCGCCTGGCCGACGAGTTGCTCTTTAGGCAGATTGCCTGGATCAAG 1198

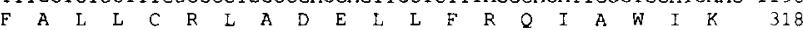
AAGCTGCCTTTCTTCTGCGAGCTCTCAATCAAGGATTACACGTGCCTCTTGAGCTCTACG 1258

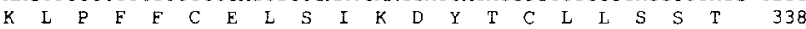
TGGCAGGAGTTAATCCTGCTCTCCTCCCTCACAGTGTACAGCAAGCAGATCTTTGGGGAG 1318 W O E L I L L S S L T V Y S K O I F G E 358 CTGGCTGATGT CACAGCCAAGTACTCACCCTCTGATGAAGAACTCCACAGATTTAGTGAT 1378 L A D V T A K Y S P S D F E I H F F S D 378 GAAGGGATGGAGGTGATTGAACGACTCATCTACCTATATCACAAGTTCCATCAGCTGAAG 1438

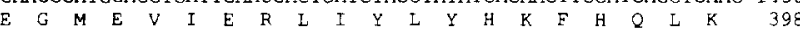
GTCAGCAACGAGGAGTACGCATGCATGAAAGCAATTAACTTCCTGAATCAAGATATCAGG 1498

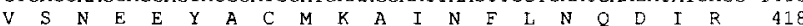
GGTCTGACCAGTGCCTCACAGCTGGAACAACTGAACAAGCGGTATTGGTACATTTGTCAG 1558

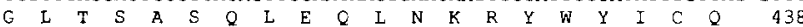
GATTTCACTGAATATAAATACACACATCAGCCAAACCGCTTTCCTGATCTTATGATGTGC 1618 $\begin{array}{lllllllllllllllllllll}D & F & T & E & Y & K & Y & T & H & O & \text { P } & \text { N } & \text { R } & \text { F } & \text { P } & \text { D } & \text { L } & \text { M } & \text { M } & \text { C } & 458\end{array}$ TTGCCAGAGATCCGATACATCGCAGGCAAGATGGTGAATGTGCCCCTGGAGCAGCTGCCC 1678 $\begin{array}{lllllllllllllllllllll}\text { I } & \text { P } & \text { E } & \text { I } & R & Y & I & \text { A } & G & \text { K } & \text { M } & \text { V } & \text { N } & \text { V } & \text { P } & \text { L } & \text { E } & \text { Q } & \text { L } & \text { P } & 478\end{array}$ CTCCTCTTTAAGGTGGTGCTGCACTCCTGCAAGACAAGTACGGTGAAGGAGTGA 1732 CTGIGCCCTGCACCTCCTTGGGCCACCCACAGTGCCTTGGGTAGGCAGCACAGGCTCCAG AGGAAAGAGCCAGAGACCAAGATGGAGACTGTGGAGCAGCTACCICCATCACAAGAAGAA

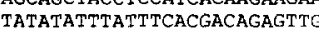
AATGTATGGCCTTCAACATGATGACATCCTTTTGTGTGAATGCAGCAGATGCATrTTCCT TGCAGTTTACAGAATGTGAAGATGTTTAATGTTA

Fig. 1. Nucleotide sequence of RTR and deduced a sequence. The putative start codon is at $\mathrm{nt} 245$. DNA-binding domain is underlined. The stop codon (nt 1730) at the end of RTR aa sequence is indicated as *. This sequence data have been deposited with EMBL/GenBank Data Libraries under accession No. U09563. Methods: (a) PCR amplification: A set of degenerate primers were designed according to the most highly conserved sequence of the DNA-binding domain of members of the human nuclear receptor family (Laudet et al., 1992). The sense-strand primer R-P1 was 5'-TGYGARGGNTGYAARGGYTTYTT-3'; (CEGCKGFF).

\section{EXPERIMENTAL AND DISCUSSION}

\section{(a) Cloning of RTR cDNA}

Highly degenerate primers were designed according to the two best-conserved aa sequences present in the DNAbinding domain of members of the nuclear receptor family. These primers were then employed in amplification reactions with single-stranded cDNA from human lung poly $(\mathrm{A})^{+}$RNA in order to identify DNA-binding domains of novel nuclear receptors. The amplified fragments of the expected size $(130 \mathrm{bp})$ were cloned and sequenced. Most of the cDNA sequences encoded DNAbinding domains of known receptors including those of RAR $\alpha$ (Petkovich et al., 1987; Giguere et al., 1987), RXR $\beta$ (Yu et al., 1991), RZR $\alpha$ (Becker-Andre et al., 1993), COUP-TF (Wang et al., 1989) and PPAR $\gamma$ (Zhu et al., 1993). Among these cDNAs, we identified one which encoded a unique sequence not previously described. The aa predicted by this cDNA sequence suggested that it encoded the DNA-binding domain of a novel member of the nuclear receptor superfamily which we refer to as RTR. With the help of the 5 -RACE method we obtained a 280 -bp fragment of the $R T R$ sequence that was subsequently used as a probe in screening a mouse testis cDNA library. After screening $3 \times 10^{5}$ independent plaques, we obtained 4 positive clones. One of these clones contained an insert of $2006 \mathrm{bp}$. Analysis of this RTR sequence

The antisense strand primer R-P2 was 5'-RCAYTTCTKNCGYCAGTAYTGRCA-3' (CQYCRL(K/Q)KC), where $\mathrm{K}=\mathrm{G}$ or $\mathrm{T}, \mathrm{N}=\mathrm{A}$ or $C$ or $G$ or $T, R=A$ or $G$ and $Y=C$ or $T$. Human lung poly $(A)^{+} R N A$ (Clontech, San Diego, CA, USA) was reverse transcribed into single strand cDNA with Mu-MLV reverse transcriptase and oligo d(T) primer (Stratagene, La Jolla, CA, USA). The R-P1 and R-P2 primers were employed in amplification reactions using the Amplitaq kit and a DNA thermal cycler (Perkin-Elmer Cetus, Norwalk, CT, USA). We carried out the following amplification cycles; 1 min denaturation at $94 \mathrm{C}$ followed by a $1 \mathrm{~min}$ annealing at $50^{\circ} \mathrm{C}$ and $3 \mathrm{~min}$ extension at $72 \mathrm{C}$ for 30 cycles. The amplification products were separated on a $2 \%$ low temparature melting agarose gel (Sigma, St. Louis, MO, USA) and the DNA products around $130 \mathrm{bp}$ were isolated from the gel and directly ligated into the TA cloning vector (Invitrogen, San Diego, CA, USA). The DNA inserts of 30 clones were analyzed by double-strand dideoxy DNA sequencing using Sequenase (US Biochemical, Cleveland, $\mathrm{OH}$, USA). (b) Anchor PCR: To isolate a greater region of the gene encoding RTR, we used the 5'-RACE kit (BRL) in the same manner as described previously (Loh et al., 1989). Two sequential antisense primers were designed from the sequence of the original RTR PCR fragment Single-stranded cDNA primed with specific primer was synthesized from $1 \mu \mathrm{g}$ of human lung poly $(\mathrm{A})^{+} \mathrm{RNA}$ with Superscript reverse transcriptase according to manufacturer's recommendation (BRL). The amplification products were cloned into pBluescript II and sequenced (c) cDNA library screening: Uni-ZAP XR cDNA libraries from mouse testis (Stratagene) were screened with multiprimed cDNA probe from anchor PCR products with moderately stringent conditions. cDNA inserts from several positive clones were subcloned into the EcoRI and Xhol site of pBluescript II. The complete DNA sequences of both strands were determined as described for $\mathrm{PCR}$ products. 
revealed a long open reading frame which starts with a putative start codon at nt 245 and terminates with a putative stop codon at 1730 (Fig. 1). On this basis, RTR encodes a protein of 495 aa with a predicted molecular mass of $60 \mathrm{kDa}$. The DNA-binding domain (aa 75-140) of RTR contains 10 Cys residues, eight of which participate in the formation of two zinc-finger motifs.

\section{(b) Homologies of RTR with other nuclear receptors}

Comparison of aa sequence of RTR with those of other members of nuclear receptor superfamily revealed that RTR does not belong to any of the subfamilies of nuclear receptors reported previously. RTR exhibits a $32-34 \%$ overall identity with the mouse RXR receptors (Mangelsdorf et al., 1992; Leid et al., 1992). The DNAbinding domain of RTR showed the highest identity $(61 \%)$ with that of the mouse RXR, the human RZR $\alpha$ and mouse $\operatorname{RAR} \gamma$ receptors (Fig. 2a). The P-box sequence which constitutes the aa sequence present in the C-terminal region of the first zinc-finger is key in classifying the DNA-binding specificity of nuclear receptors (Forman and Samuels, 1990). In RTR, this sequence is
CEGCKG, indicating that RTR may recognize enhancer sequences similar to those of the retinoid, thyroid hormone, vitamin $\mathrm{D}$ and peroxisome proliferator activating receptors (Laudet et al., 1992). Comparison of the aa sequence of the ligand-binding domain indicated little homology between RTR and other nuclear receptors (Fig. 2b). The highest identity ( $26 \%$ ) was found between the ligand binding domain of RTR and that of mouse COUP-TFII (Qiu et al., 1994). The ligand-binding domain of mouse $\operatorname{RXR} \propto$ exhibited a $20 \%$ identity with that of RTR.

\section{(c) Tissue distribution of RTR mRNA}

To study the tissue distribution of RTR mRNA, we performed Northern blot analysis on poly $(\mathrm{A})^{+}$RNA from a variety of mouse tissues (Fig. 3). The full-length $R T R$ probe hybridized to a $7.4-\mathrm{kb}$ and a $2.3-\mathrm{kb}$ transcript in testis. The 7.4-kb transcript was much less in brain, lung, liver and kidney than testis and the 2.3-kb transcript was not detected. The expression of transcripts of distinct size has been reported for several other orphan receptors. For example, the orphan receptor RZR $\alpha$ has a $15-\mathrm{kb}$

a.

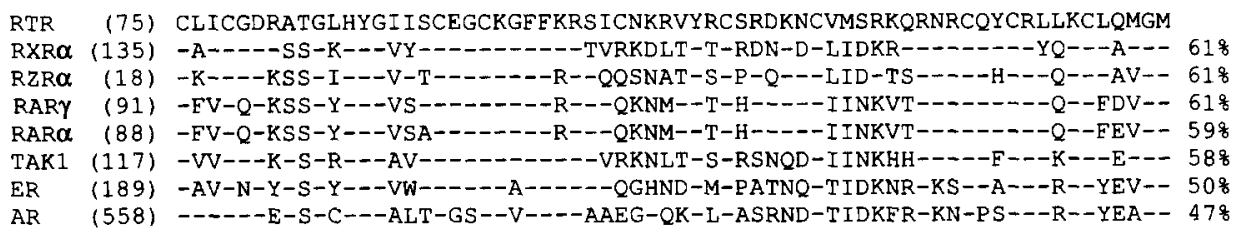

b.

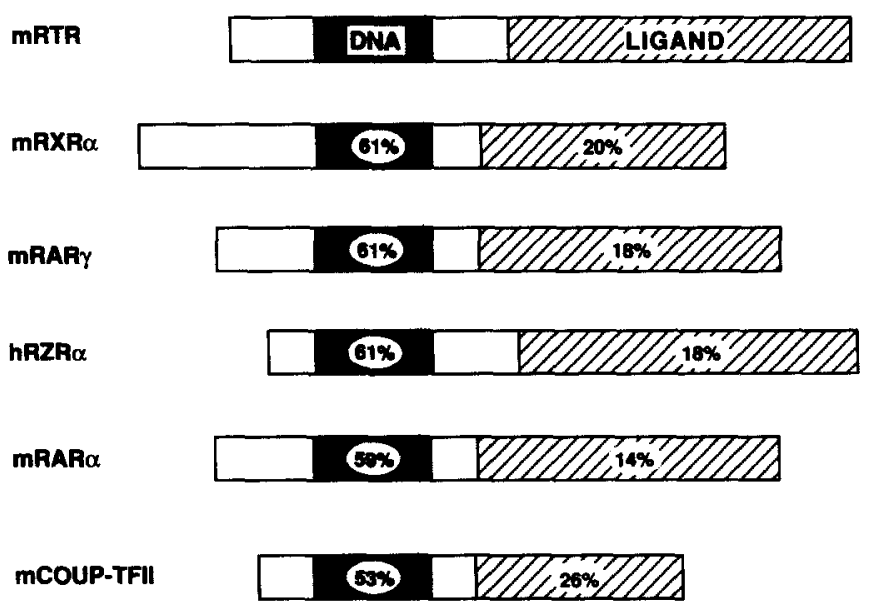

Fig. 2. Schematic aa comparison of RTR and several members of the steroid hormone receptor superfamily. (a) The aa sequence alignment of the DNA-binding domain of RTR and members of the steroid hormone receptor superfamily. The number in parentheses represent the positions of aa residues in the individual receptors. The aa conserved with RTR are indicated with a bar. The numbers on the right of aa sequences indicate the percentage identity with RTR. (b) Alignment of aa sequences of DNA-binding domain and ligand-binding domain. The numbers in each box indicate the percentage of identity with RTR. The DNA- and ligand-binding domains are indicated. Specific references are mRXR (Mangelsdorf et al., 1992), mRAR $\gamma$ (Zelent et al., 1989), hRZR $\alpha$ (Becker-Andre et al., 1993), mRAR $\alpha$ (Zelent et al., 1989), mCOUP-TFII (Qiu et al., 1994), hTAK1 (Hirose et al., 1994), estrogen receptor (mER, White et al., 1987) and androgen receptor (hAR, Lubahn et al., 1988). The prefixes $h$ and $m$ denote human and mouse, respectively. 


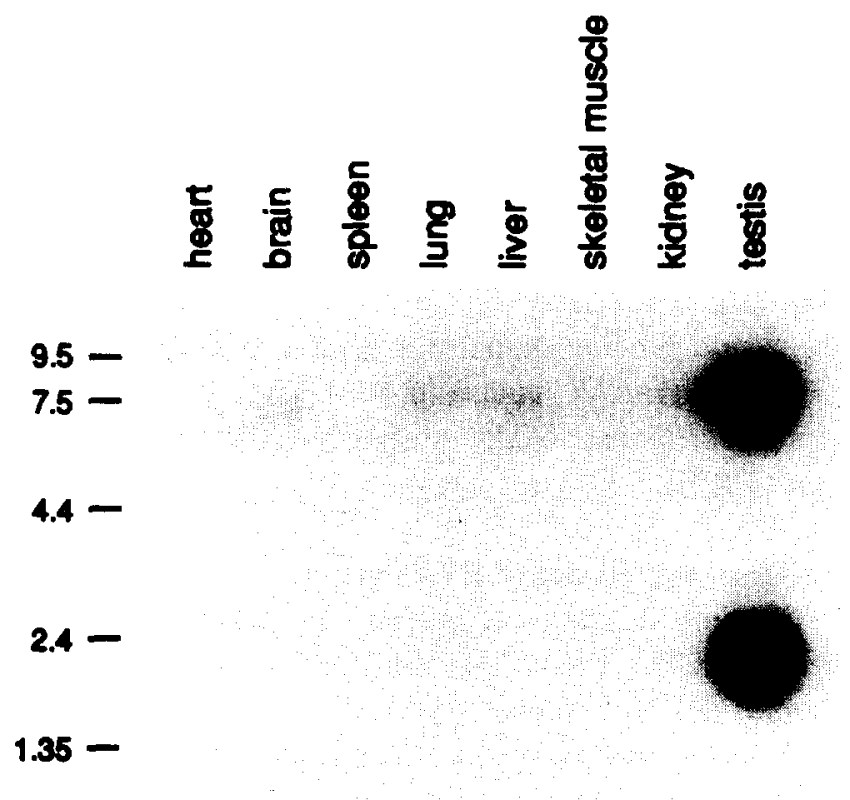

Fig. 3. Tissue specificity of $R T R$ expression. Poly $(\mathrm{A})^{+} \mathrm{RNA}(2 \mu \mathrm{g})$ isolated from different Balb/c mouse tissues was analyzed by Northern blot analysis using a ${ }^{32} \mathrm{P}$-labeled $R T R$ probe. RNA size markers $(\mathrm{kb})$ are indicated on the left side of blot. Full-length $\mathrm{CDNA}$ of $R T R$ labeled with ${ }^{32} \mathrm{P}$ by random priming was used to detect $R T R$ mRNA. Methods: Mouse multiple tissue Northern blots $(1.2 \%$ agarose- $2.2 \mathrm{M} \mathrm{HCOH})$ were purchased from Clontech. The blots were hybridized with a ${ }^{32} \mathrm{P}$ labeled $R T R$ probe encoding nt 300 to 2014 as described previously (Hirose et al., 1990).

transcript that is expressed ubiquitously and a $2.3-\mathrm{kb}$ transcript expressed predominantly in leukocytes, testis, lung and liver (Becker-Andre et al., 1993). The unrelated orphan receptor TAK1 (Fig. 2a) which exhibits $26 \%$ identity with RTR has also two distinct transcripts, with the shorter one specifically expressed in testis (Hirose et al., 1994). The different transcripts of TAK1 and RTR may be generated by alternative splicing.

\section{(d) Cell-type-specific expression of $R T R$ in mouse testis}

Mature testis is composed of many cell types, including Sertoli cells, Leydig cells and a variety of germ cells (Russel et al., 1990). To investigate which cells in the testis expressed $R T R$, we performed Northern blot analysis using mRNA prepared from different testicular cells (Fig. 4). The full-length RTR cDNA probe hybridized to mRNA from whole testis and mixed germ cells but not to mRNA from Sertoli cells (Fig. 4a). The ratio between the 2.3 and $7.4-\mathrm{kb}$ transcripts in testis of CD1 mice was different from that of Balb/c mice. Mixed germ cells contain spermatogonia, several kinds of spermatocytes and haploid spermatids. Therefore, mixed germ cells were separated into distinct cell populations and subsequently analyzed for $R T R$ expression (Fig. 4b). RTR mRNA was highly expressed in round spermatids and was not detectable in germ cells at an earlier phase of spermatogenesis.

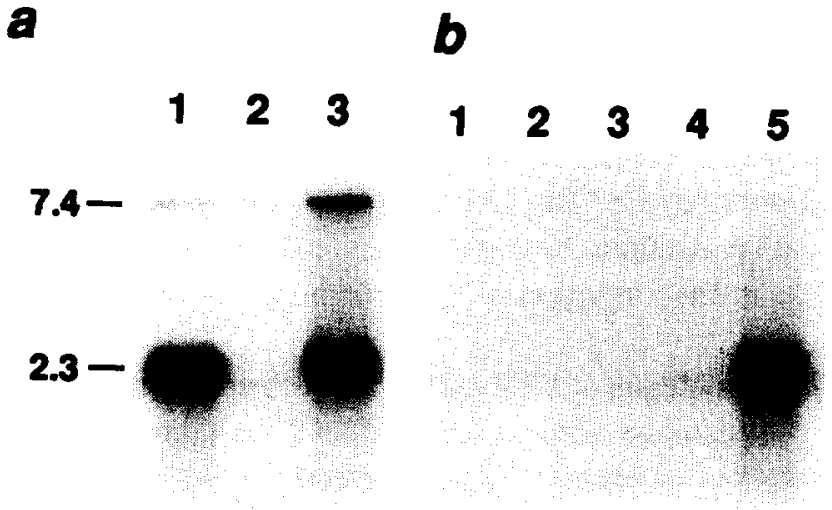

Fig. 4. Specificity of $R T R$ expression in testis. Poly(A) ${ }^{+}$RNA $(8 \mu \mathrm{g})$ isolated from different testicular cells was analyzed by Northern blot analysis using a ${ }^{32} \mathrm{P}$-labeled $R T R$ probe. (a) mRNA from whole testis (1), Sertoli cells (2) and mixed germ cells (3). (b) mRNA from different germ cells; mRNA from type B spermatogonia (1), preleptotene spermatocytes (2), leptotene- and zygotene spermatocytes (3), pachytene spermatocytes (4) and round spermatids (5). Equal amounts of RNA were loaded as determined by residual ribosomal RNA content. Methods: Mixed germ cell suspensions were prepared from adult CD-1 mice by enzymatic dissociation of seminiferous tubules as described previously (O'Brien, 1987). Germ cells at defined stages of differentiation were isolated from mixed cell suspensions by unit gravity sedimentation (Bellve et al., 1977; O'Brien, 1987). Sertoli cells from 17-day-old CD-1 mice were isolated and maintained in culture for 7 days as described previously (O'Brien et al., 1989). Northern blot analysis (1.0\% agarose$2.2 \mathrm{M} \mathrm{HCOH}$ ) was performed as described in the legend to Fig. 3.

These observations indicate that $R T R$ transcripts are most abundant in haploid germ cells and suggest that RTR may regulate gene expression in particular during the post-meiotic phase of spermatogenesis. A number of other genes have been reported to be up-regulated during the post-meiotic phase of spermatogenesis, including protamines (Kleene et al., 1983), the proto-oncogenes c- $a b l$ and c-mos (Iwaoki et al., 1993), and glyceraldehyde 3-phosphate dehydrogenase (Mori et al., 1992). Some of these genes may be candidates for regulation by RTR.

\section{(e) Conclusions}

(1) A cDNA encoding a novel member of the nuclear receptor superfamily was isolated. The deduced aa sequence of the new receptor, referred to as RTR, exhibited the highest identity (32\%) with members of the RXR family.

(2) $R T R$ is predominantly expressed in the testis and at very low levels in lung, brain, kidney and liver.

(3) RTR mRNA was most highly expressed in round spermatids, suggesting a role for RTR in the regulation of gene expression during the post-meiotic phase of spermatogenesis.

\section{ACK NOWLEDGEMENTS}

This work was partially supported by HD26485 (D.A.O.), P30-HD18968 (The Laboratories for 
Reproductive Biology) and CA16086 (UNC Lineberger Cancer Center) grants from NIH. The authors thank Drs. E.M. Eddy, K. Korach and D.J. Dix for their comments on the manuscript.

\section{REFERENCES}

Becker-Andre, M., Andre, E. and DeLamarter, J.F.: Identification of nuclear receptor mRNAs by RT-PCR amplification of conserved zinc-finger motif sequences. Biochem. Biophys. Res. Commun. 194 (1993) 1371-1379.

Bellve, A.R., Millette, C.F., Bhatnagar, Y.M. and O'Brien, D.A.: Dissociation of the mouse testis and characterization of isolated spermatogenic cells. J. Histochem. Cytochem. 25 (1977) 480-494.

Evans, R.M.: The steroid and thyroid receptor superfamily. Science 240 (1988) 889-895.

Forman, B.M. and Samuels, H.H.: Interaction among a subfamily of nuclear hormone receptors: the regulatory zipper model. Mol. Endocrinol. 4 (1990) 1293-1301.

Giguere, V., Ong, E.S., Segui, P. and Evans, R.M.: Identification of a receptor for the morphogen retinoic acid. Nature 330 (1987) 624-629.

Green, S. and Chambon, P.: Nuclear receptors enhance our understanding of transcriptional regulation. Trends Genet. 4 (1988) 309-314.

Hirose, T., Koga, M., Kouhara, H., Kishimoto, S., Matsumoto, K. and Sato, B.: Effect of retinoic acid on proliferation of estrogenresponsive transformed murine Leydig cells in serum-free culture. Cancer Res. 50 (1990) 5060-5064.

Hirose, T., Fujimoto, W., Yamaai, T., Kim, K.-H., Matsuura, H. and Jetten, A.M.: TAK1: molecular cloning and characterization of a new member of the nuclear receptor superfamily. Mol. Endocrinol. $8(1994)$ in press.

Iwaoki, Y., Matsuda, H., Mutter, G.L., Wartin, F. and Wolgemuth, D.J.: Differential expression of the proto-oncogenes c-abl and c-mos in developing mouse germ cells. Exp. Cell Res. 206 (1993) 212-219.

Kleene, K.C., Distel, R.J. and Hecht, N.B.: cDNA clones encoding cytoplasmic poly(A) ${ }^{+}$RNAs which first appear at detectable levels in haploid phase of spermatogenesis in the mouse. Dev. Biol. 98 (1983) 455-464.

Laudet, V., Hanni, C., Coll, J., Catzeflis, F. and Stehelin, D.: Evolution of the nuclear receptor gene superfamily. EMBO J. 11 (1992) 1003-1013.

Leid, M., Kastner, Lyons, R.P., Nakashatri, H., Saunders, M., Zacharewsi, T., Chen, J.-Y., Staub, A., Garnier, J.-M., Mader, S. and Chambon, P.: Purification, cloning, and RXR identity of the Hela cell factor with which RAR or TR heterodimerizes to bind target sequences efficiently. Cell 68 (1992) 377-395.

Loh, E.L., Elliot, J.F., Cwirla, S., Larier, L.L. and Davis, M.M.: Polymerase chain reaction with single-sided specificity: analysis of T cell receptor $\delta$. Science 243 (1989) 217-220.
Lubahn, D.B., Joseph, D.R., Sullivan, P.M., Willard, H.F., French, F.S. and Wilson, E.M.: Cloning of human androgen receptor complementary DNA and localization to X chromosome. Science 240 (1988) 327-331.

Mangelsdorf, D.J., Borgmeyer, U., Heyman, R.A., Zhou, J.Y., Ong, E.S., Oro, A.E., Kakizuka, A. and Evans, R.M.: Characterization of three RXR genes that mediate the action of 9-cis retinoic acid. Genes Dev. 6 (1992) 329-344.

Mori, C., Welch, J.E., Sakai, Y. and Eddy, E.M.: In situ localization of spermatogenic cell-specific glyceraldehyde 3-phosphate dehydrogenase messenger ribonucleic acid in mice. Biol. Reprod. 46 (1992) 859-868.

O'Brien, D.A.: Stage-specific protein synthesis by isolated spermatogenic cells throughout meiosis and early spermiogenesis. Biol. Reprod. 37 (1987) 147-157.

O'Brien, D.A., Gabel, C.A., Rockett, D.L. and Eddy, E.M.: Receptormediated endocytosis and differential synthesis of mannose-6-phosphate receptors in isolated spermatogenic and Sertoli cells. Endocrinology 124 (1989) 2973-2984.

Petkovich, M., Brand, N.J., Krust, A. and Chambon, P.: A human retinoic acid receptor which belongs to the family of nuclear receptors. Nature 330 (1987) 444-450.

Qiu, Y., Cooney, A.J., Kuratani, S., DeMayo, F.J., Tsai, S.Y. and Tsai, M.J.: Spatiotemporal expression patterns of chicken ovalbumin upstream promoter-transcription factors in the developing mouse central nervous system: evidence for a role in segmental patterning of the diencephalon. Proc. Natl. Acad. Sci. USA 91 (1994) 4451-4455.

Russell, L.D., Ettlin, R.A., Sinha Hikim, A.P. and Clegg, E.D.: Histological and Histopathological Evaluation of the Testis. Cache River Press, Clearwater, FL, 1990.

Wang, L.-H., Tsai, S.Y., Cook, R.G., Beattie, W.G., Tsai, M.J. and O'Malley, B.W.: COUP transcription factor is a member of the steroid receptor family. Nature 340 (1989) 156-160.

White, R., Lees, J.A., Needham, M. and Parker, M. Structural organization and expression of the mouse estrogen receptor. Mol. Endocrinol. 1 (1987) 735-744.

Yamamoto, K.R.: Steroid receptor regulated transcription of specific genes and gene networks. Annu. Rev. Genet. 19 (1985) 209-252.

Yu, V.C., Delsert, C., Andersen, B., Holloway, J.M., Devary, O.V., Naar, A.M., Kim, S.Y., Boutin, J.-M. Glass, C.K. and Rosenfeld, M.G.: RXR $\beta$ : a coregulator that enhances binding of retinoic acid, thyroid hormone, and vitamin $\mathrm{D}$ receptors to their cognate response elements. Cell 67 (1989) 1251-1266.

Zelent, A., Krust, A., Petkovich, M., Kastner, P. and Chambon, P.: Cloning of murine alpha and beta retinoic acid receptors and a novel receptor gamma predominantly expressed in skin. Nature 339 (1989) 714-717.

Zhu, Y., Alvares, K., Huang, Q., Rao, M.S. and Reddy, J.K.: Cloning of a new member of the peroxisome proliferator-activated receptor gene family from mouse liver. J. Biol. Chem. 268 (1993) $26817-26820$. 